\title{
Extremal graphs with a given number of perfect matchings
}

\author{
Stephen G. Hartke* \\ Derrick Stolee ${ }^{\dagger}$ \\ Douglas B. West $\ddagger$ \\ Matthew Yancey ${ }^{\S}$
}

May 5, 2011

\begin{abstract}
Let $f(n, p)$ denote the maximum number of edges in a graph having $n$ vertices and exactly $p$ perfect matchings. For fixed $p$, Dudek and Schmitt showed that $f(n, p)=$ $n^{2} / 4+c_{p}$ for some constant $c_{p}$ when $n$ is at least some constant $n_{p}$. For $p \leq 6$, they also determined $c_{p}$ and $n_{p}$. For fixed $p$, we show that the extremal graphs for all $n$ are determined by those with $O(\sqrt{p})$ vertices. As a corollary, a computer search determines $c_{p}$ and $n_{p}$ for $p \leq 10$. We also present lower bounds on $f(n, p)$ proving that $c_{p}>0$ for $p \geq 2$ (as conjectured by Dudek and Schmitt), and we conjecture an upper bound on $f(n, p)$. Our structural results are based on Lovász's Cathedral Theorem.
\end{abstract}

\section{Introduction}

For even $n$ and positive integer $p$, Dudek and Schmitt [5] defined $f(n, p)$ to be the maximum number of edges in an $n$-vertex graph having exactly $p$ perfect matchings. Say that such a graph with $f(n, p)$ edges is $p$-extremal. We study the behavior of $f(n, p)$ and the structure of $p$-extremal graphs.

*Department of Mathematics, University of Nebraska-Lincoln, hartke@math.unl.edu. Research supported by a Nebraska EPSCoR First Award and by National Science Foundation grant DMS-0914815.

${ }^{\dagger}$ Departments of Mathematics and Computer Science, University of Nebraska-Lincoln, s-dstolee1@math.unl.edu. Research supported by National Science Foundation grants CCF-0916525 and DMS-0914815.

${ }^{\ddagger}$ Department of Mathematics, University of Illinois, Urbana, IL, west@math.uiuc.edu. Research supported by NSA Award H98230-10-1-0363.

$\S$ Department of Mathematics, University of Illinois, Urbana, IL, yancey1@illinois.edu. Research supported by NSF grant DMS 08-38434, "EMSW21-MCTP: Research Experience for Graduate Students". 
Although existence of a perfect matching can be tested in time $O\left(n^{1 / 2} m\right)$ for graphs with $n$ vertices and $m$ edges [14], counting the perfect matchings is \#P-complete, even for bipartite graphs [19]. Let $\Phi(G)$ denote the number of perfect matchings in $G$. Bounds on $\Phi(G)$ are known in terms of the vertex degrees in $G$. For a bipartite graph $G$ with $n$ vertices in each part and degrees $d_{1}, \ldots, d_{n}$ for the vertices in one part, Brègman's Theorem [2] states that $\Phi(G) \leq \prod_{i=1}^{n}\left(d_{i} !\right)^{1 / d_{i}}$. Kahn and Lovász (unpublished) proved an analogue for general graphs (other proofs were given by Friedland [6] and then by Alon and Friedland [1]). For a graph $G$ with vertex degrees $d_{1}, \ldots, d_{n}$, the Kahn-Lovász Theorem states that $\Phi(G) \leq \prod_{i=1}^{n}\left(d_{i} !\right)^{1 / 2 d_{i}}$. Both results were reproved using entropy methods by Radhakrishnan [16] and by Cutler and Radcliffe [4], respectively. Gross, Kahl, and Saccoman [7] studied $\Phi(G)$ for a fixed number of edges; they determined the unique graphs minimizing and maximizing $\Phi(G)$.

Maximizing the number of edges when $\Phi(G)$ and $n$ are fixed has received less attention. Hetyei proved that $f(n, 1)=n^{2} / 4$ (see [11, Corollary 5.3.14, page 173]). We describe Hetyei's construction inductively in a more general context.

Construction 1.1. The Hetyei-extension of $G$ is the graph $G^{\prime}$ formed from $G$ by adding a vertex $x$ adjacent to all of $V(G)$ and one more vertex $y$ adjacent only to $x$. Every perfect matching of $G^{\prime}$ contains $x y$ and a perfect matching of $G$, so $\Phi\left(G^{\prime}\right)=\Phi(G)$. Starting with $G=K_{2}$, Hetyei-extension yields graphs with one perfect matching for all even orders.

When $G$ has $n$ vertices, $\left|E\left(G^{\prime}\right)\right|=|E(G)|+n+1$. Since $(k+2)^{2} / 4=k^{2} / 4+k+1$, we obtain $f(n, 1) \geq n^{2} / 4$ for all even $n$. (Note that when $G$ has a unique perfect matching $M$, at most two edges join the vertex sets of any two edges of $M$; hence $f(n, 1) \leq n / 2+2\left(\begin{array}{c}n \\ 2\end{array}\right)=n^{2} / 4$. $)$

More generally, when $\Phi(G)=p$ and $|E(G)|=n^{2} / 4+c$, the Hetyei-extension of $G$ yields $f(n+2, p) \geq(n+2)^{2} / 4+c$. This observation is due to Dudek and Schmitt [5].

In light of the observation in Construction 1.1, we let $c(G)=|E(G)|-|V(G)|^{2} / 4$ and call $c(G)$ the excess of $G$. For fixed $p$, Dudek and Schmitt proved that the maximum excess is bounded by a constant.

Theorem 1.2 (Dudek and Schmitt [5]). For $p \in \mathbf{N}$, there is an integer $c_{p}$ and a threshold $n_{p}$ such that $f(n, p)=n^{2} / 4+c_{p}$ when $n \geq n_{p}$ and $n$ is even. Also, $-(p-1)(p-2) \leq c_{p} \leq p$.

Dudek and Schmitt determined $c_{p}$ and $n_{p}$ for $1 \leq p \leq 6$, although the proofs for $p \in$ $\{5,6\}$ were omitted since they were prohibitively long. They conjectured that $c_{p}>0$ when $p \geq 2$. We prove their conjecture in Section 2 by generalizing Hetyei's construction. The construction yields $c_{p}>0$ but does not generally give the best lower bounds. We give better lower bounds in Section 7; first we must analyze the structure of extremal graphs.

We develop a systematic approach to computing $c_{p}$. With this we give shorter proofs for $p \leq 6$ and identify the values $c_{p}$ and $n_{p}$ for $7 \leq p \leq 10$. Using structural results from this 
paper and further computational techniques, $c_{p}$ and $n_{p}$ are determined for $p \leq 27$ in [17]. The complete behavior of $c_{p}$ for larger $p$ remains unknown.

Definition 1.3. Let $\mathcal{F}_{p}$ denote the family of graphs that are $p$-extremal and have excess $c_{p}$; that is, $\mathcal{F}_{p}=\left\{G: \Phi(G)=p\right.$ and $\left.|E(G)|=\frac{|V(G)|^{2}}{4}+c_{p}\right\}$. Equivalently, $\mathcal{F}_{p}$ is the set of $p$-extremal graphs with at least $n_{p}$ vertices.

We study the extremal graphs as a subfamily of a larger family.

Definition 1.4. A graph is saturated if the addition of any missing edge increases the number of perfect matchings.

Extremal graphs are contained in the much larger family of saturated graphs. Figure 1(a) shows a saturated graph $G_{1}$ with 12 vertices, eight perfect matchings, and 27 edges. Although $G_{1}$ is saturated, it is not 8-extremal, since the graph $G_{2}$ in Figure 1 (b) has the same number of vertices and perfect matchings but has 39 edges.

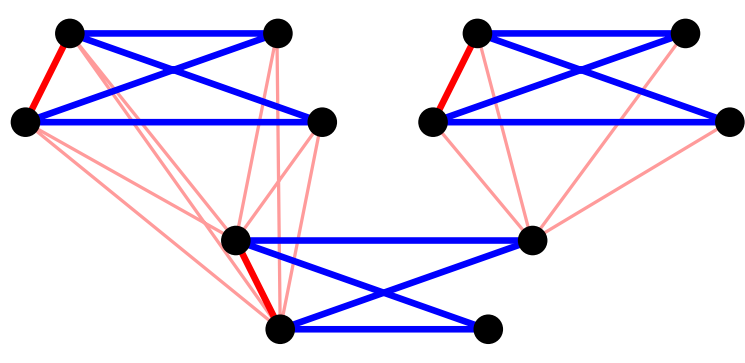

(a) $G_{1}$ with 27 edges

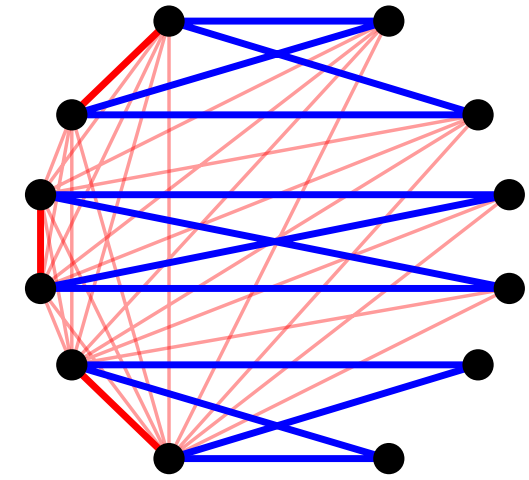

(b) $G_{2}$ with 39 edges

Figure 1: Two graphs with eight perfect matchings

Lovász's Cathedral Theorem (see [11]) gives a recursive decomposition of all saturated graphs; we describe it in Section 3. In terms of this construction, we describe the graphs in $\mathcal{F}_{p}$. In Sections 4 and 5 , study of the cathedral construction for extremal graphs allows us to reduce the problem of computing $c_{p}$ to examining a finite (but large) number of graphs.

In Section 6 , we extend $f(n, p)$ to odd $n$ and study the corresponding extremal graphs. Section 7 gives constructions for improved lower bounds on $c_{p}$. In Section 8, we conjecture an upper bound on $c_{p}$ that would be sharp for infinitely many values of $p$. The conjectured bound would be the best possible monotone upper bound, if true. Section 9 mentions several conjectures and discusses a computer search based on our structural results; the search found the extremal graphs for $4 \leq p \leq 10$. Other search techniques are used in [17] to determine $c_{p}$ for $p \leq 27$. 


\section{The Excess is Positive}

We begin with a simple construction proving the Dudek-Schmitt conjecture that $c_{p}>0$.

The disjoint union of graphs $G$ and $H$ (with disjoint vertex sets) is denoted $G+H$. The join of $G$ and $H$, denoted $G \vee H$, consists of $G+H$ plus edges joining each vertex of $G$ to each vertex of $H$. Thus the Hetyei-extension of $G$ is $\left(G+K_{1}\right) \vee K_{1}$. A split graph is a graph whose vertex set is the union of a clique and an independent set.

Definition 2.1. The Hetyei graph with $2 k$ vertices, produced iteratively in Construction 1.1 from $K_{2}$ by repeated Hetyei-extension, can also be described explicitly. It is the split graph with clique $\ell_{1}, \ldots, \ell_{k}$, independent set $r_{1}, \ldots, r_{k}$, and additional edges $\ell_{i} r_{j}$ such that $i \leq j$.

The Hetyei graph is the unique extremal graph of order $2 k$ with exactly one perfect matching. It has $\frac{(2 k)^{2}}{4}$ edges, so $c_{1}=0$. In the constructions here and in Section 7 , the Hetyei graph is a proper subgraph, so the excess is larger.

In a graph having an independent set $S$ with half the vertices, every perfect matching joins $S$ to the remaining vertices. Therefore, to study the perfect matchings in such a graph it suffices to consider the bipartite subgraph consisting of the edges incident to $S$. In the Hetyei graph, the only perfect matching consists of the edges $\ell_{i} r_{i}$ for all $1 \leq i \leq k$.

For $m \in \mathbf{N}$, let $w(m)$ denote the number of 1 s in the binary expansion of $m$.

Definition 2.2. For $p \geq 2$ and $k=\left\lceil\log _{2}(p-1)\right\rceil+1$, let $\left(x_{k-2}, \ldots, x_{0}\right)$ be the binary $(k-1)$ tuple such that $p-1=\sum_{j=0}^{k-2} 2^{j} x_{j}$. The binary expansion construction for $p$, denoted $B(p)$, consists of the Hetyei graph with $2 k$ vertices plus the edges $\left\{\ell_{i+2} r_{1}: x_{i}=1\right\}$ (see Fig. 2).

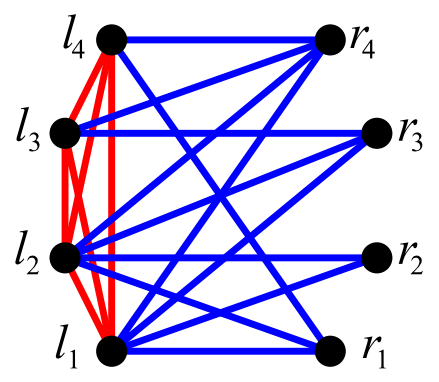

Figure 2: The graph $B(6)$

Theorem 2.3. If $p \geq 2$, then $\Phi(B(p))=p$ and $c(B(p))=w(p-1)$. Thus $c_{p} \geq w(p-1) \geq 1$.

Proof. Name the vertices of $B(p)$ as in the Hetyei graph. We construct perfect matchings in $B(p)$ by successively choosing the edges that cover $r_{1}, \ldots, r_{k}$. The matching $\left\{\ell_{i} r_{i}: 1 \leq i \leq k\right\}$ 
from the Hetyei graph is always present. If $r_{1}$ is matched to $\ell_{i+2}$ instead of to $\ell_{1}$ for some nonnegative $i$, then for $r_{2}, \ldots, r_{i-1}$ exactly two edges are available when we choose the edge to cover this vertex. For vertices $r_{i}, \ldots, r_{k}$ in order, only one choice then remains. Therefore, each edge of the form $\ell_{i+2} r_{1}$ lies in $2^{i-2}$ perfect matchings.

The edge $\ell_{i+2} r_{1}$ exists if and only if $x_{i}=1$ in the binary representation of $p-1$. Thus $\Phi(B(p))=1+\sum_{i=2}^{k} 2^{i-2} x_{i-2}+1=1+p-1=p$. Since $B(p)$ is formed by adding $w(p-1)$ edges to the Hetyei graph, $c(B(p))=w(p-1)$.

\section{Lovász's Cathedral Theorem}

As we have mentioned, Lovász's Cathedral Theorem characterizes saturated graphs. Since the extremal graphs are saturated, this characterization will be our starting point. Chapters 3 and 5 of Lovász and Plummer [11] present a full treatment of the subject. Another treatment appears in $\mathrm{Yu}$ and Liu [20]. A 1-factor of a graph $G$ is a spanning 1-regular subgraph; its edge set is a perfect matching. An edge is extendable if it appears in a 1-factor.

Definition 3.1. A graph is matchable if it has a perfect matching. The extendable subgraph of a matchable graph $G$ is the union of all the 1-factors of $G$. An induced subgraph $H$ of $G$ is a chamber of $G$ if $V(H)$ is the vertex set of a component of the extendable subgraph of $G$.

Every vertex of a matchable graph $G$ is incident to an extendable edge, so the chambers of $G$ partition $V(G)$. Perfect matchings in $G$ are formed by independently choosing perfect matchings in the chambers of $G$.

Lemma 3.2. If a matchable graph $G$ has chambers $H_{1}, \ldots, H_{k}$, then $\Phi(G)=\prod_{i=1}^{k} \Phi\left(H_{i}\right)$.

The chambers form the outermost decomposition in Lovász's structure (see Fig. 3). When the extendable subgraph is connected, there is only one chamber and no further breakdown.

Definition 3.3. A graph is elementary if it is matchable and its extendable subgraph is connected.

Tutte [18] characterized the matchable graphs. An odd component of a graph $H$ is a component having an odd number of vertices; $o(H)$ denotes the number of odd components. An obvious necessary condition for existence of a perfect matching in $G$ is that $o(G-S) \leq|S|$ for all $S \subseteq V(G)$. Tutte's 1-Factor Theorem states that this condition is also sufficient.

Definition 3.4. A barrier in a matchable graph $G$ is a set $X \subseteq V(G)$ with $o(G-X)=|X|$.

Lemma 3.5 (Lemma 5.2.1 [11]). If $G$ is elementary, then the family of maximal barriers in $G$ is a partition of $V(G)$, denoted $\mathcal{P}(G)$. 


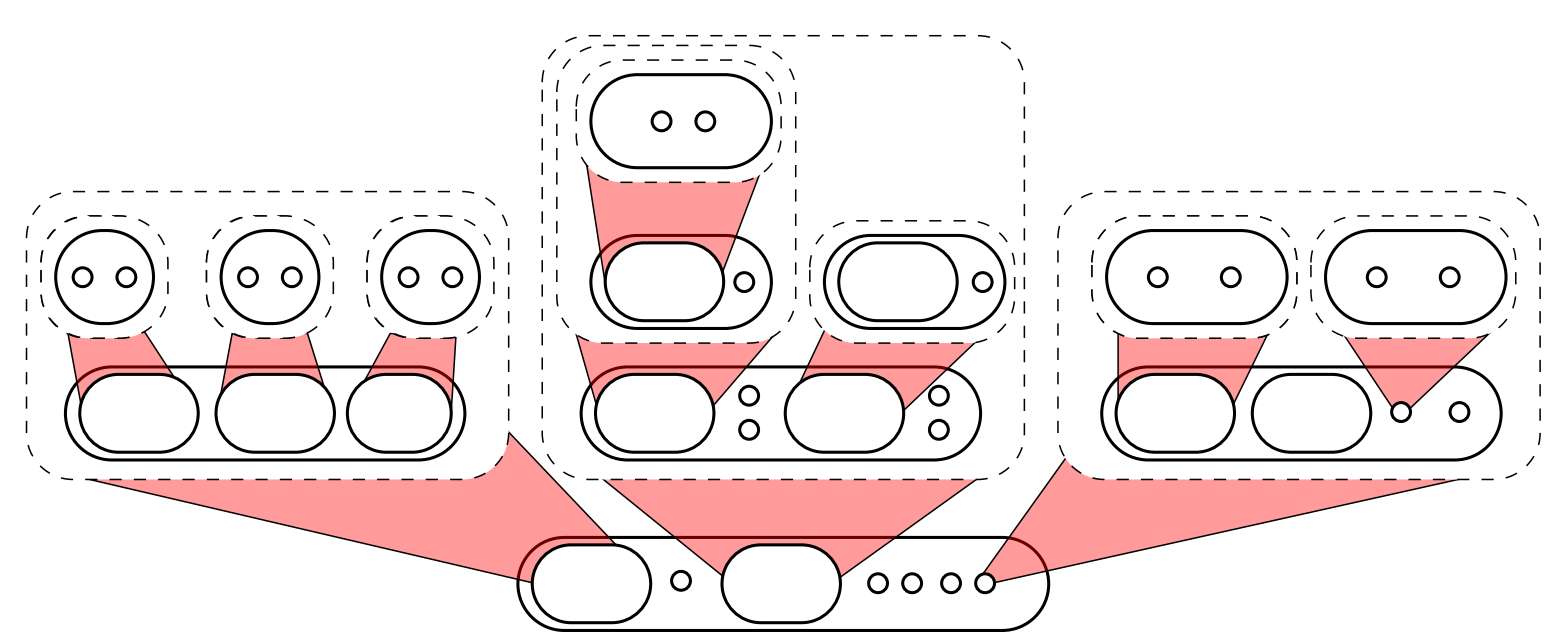

Figure 3: An example cathedral construction.

Construction 3.6 (The Cathedral Construction). A graph $G$ is a cathedral if it consists of (1) a saturated elementary graph $G_{0},(2)$ disjoint cathedrals $G_{1}, \ldots, G_{t}$ corresponding to the maximal barriers $X_{1}, \ldots, X_{t}$ of $G_{0}$, and (3) edges joining every vertex of $X_{i}$ to every vertex of $G_{i}$, for $1 \leq i \leq t$. The graph $G_{0}$ is the foundation of the cathedral. The cathedral $G_{i}$ may have no vertices when $i>0$; thus every saturated elementary graph is a cathedral (with empty cathedrals over its barriers).

Since the cathedral construction has a cathedral "above" each maximal barrier of $G_{0}$, the construction is recursive, built from saturated elementary graphs. Each nonempty subcathedral $G_{i}$ contains a saturated elementary graph $G_{i, 0}$, and each maximal barrier $X_{i, j} \in \mathcal{P}\left(G_{i, 0}\right)$ has a cathedral $G_{i, j}$ over it in $G_{i}$. Figure 3 illustrates the cathedral construction. Here cathedrals are indicated by dashed curves (except for the full cathedral). Each foundation is indicated by a solid curve, as are the barriers within it.

Theorem 3.7 (The Cathedral Theorem; Theorem 5.3 .8 [11]). A graph $G$ is saturated if and only if it is a cathedral. The foundation $G_{0}$ in the cathedral construction of $G$ is unique, and every perfect matching in $G$ contains a perfect matching of $G_{0}$.

Since each perfect matching in a cathedral $G$ contains a perfect matching of $G_{0}$, the edges joining $G_{0}$ to the cathedrals $G_{1}, \ldots, G_{t}$ appear in no perfect matching. Therefore, $G_{0}$ is a chamber in $G$. Recursively, the foundations of the subcathedrals are the chambers of $G$.

The saturated graphs of Figure 1 are cathedrals having the same chambers (and hence the same number of perfect matchings). Their cathedral structures are shown in Figure 4.

Let $G \in \mathcal{F}_{p}$ be a $p$-extremal graph. Since $G$ is extremal, it is saturated, and hence it is a cathedral. Recall that the Hetyei-extension of $G$ is $\left(G+K_{1}\right) \vee K_{1}$. The complete graph $K_{2}$ 


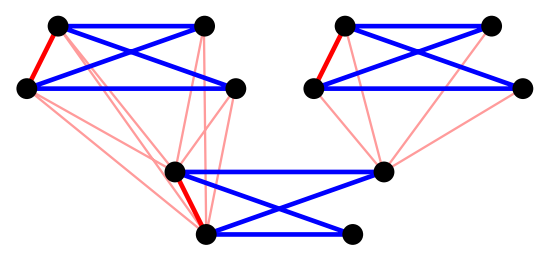

(a) $G_{1}$ with 27 edges

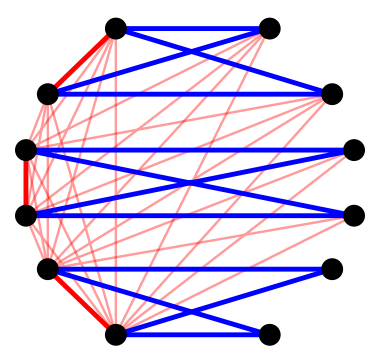

(c) $G_{2}$ with 39 edges

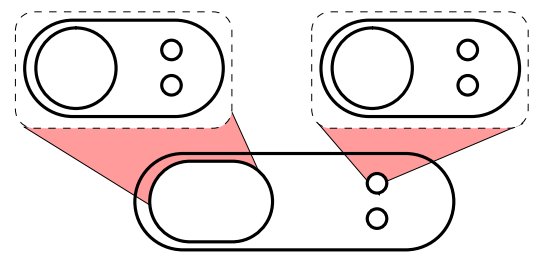

(b) $G_{1}$ cathedral

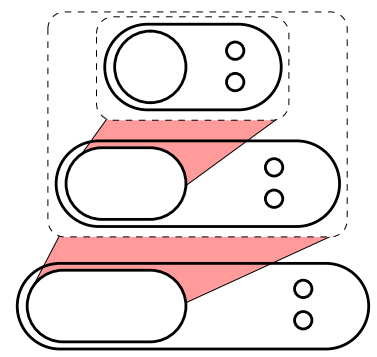

(d) $G_{2}$ cathedral

Figure 4: The saturated graphs from Figure 1 and their cathedral structures.

is a saturated elementary graph; its barriers are single vertices, say $\left\{x_{1}\right\}$ and $\left\{x_{2}\right\}$. Letting $G_{1}=G$ and $G_{2}=\varnothing$, we obtain the Hetyei-extension of $G$ as a cathedral with $G_{0}=K_{2}$.

\section{Extremal Graphs are Spires}

From the cathedral structures of the two graphs in Figure 4, it is easy to see why $G_{2}$ has many more edges. Nesting of cathedrals generates many edges from foundations to the cathedrals over them. We introduce a special term for cathedrals formed in this way.

Definition 4.1. A spire is a cathedral in which at most one maximal barrier in the foundation has a nonempty cathedral over it, and that nonempty cathedral (if it exists) is a spire. In particular, every saturated elementary graph is a spire.

In Figure 4, the graph $G_{2}$ is a spire, while $G_{1}$ is not. By the recursive definition, the chambers of a spire $G$ form a list $\left(H_{0}, \ldots, H_{k}\right)$ such that each $H_{i}$ is the foundation of the spire induced by $\bigcup_{j=i}^{k} V\left(H_{j}\right)$, and in $H_{i}$ with $i<k$ there is a maximal barrier $Y_{i}$ that is adjacent to the vertices of the spire induced by $\bigcup_{j=i+1}^{k} V\left(H_{i}\right)$. We then say that $G$ is a spire generated by $H_{0}, \ldots, H_{k}$ over $Y_{0}, \ldots, Y_{k}$.

Our first goal is to prove that extremal graphs are spires.

Lemma 4.2. Every p-extremal graph is a spire such that in each chamber, the maximal barrier having neighbors in later chambers is a barrier of maximum size. 
Proof. Since a $p$-extremal graph is saturated, it is a cathedral. Let $G$ be a cathedral having nonempty cathedrals $G_{i}$ and $G_{j}$ over maximal barriers $X_{i}$ and $X_{j}$ in its foundation, with $\left|X_{i}\right| \geq\left|X_{j}\right|$. Let $G^{\prime}$ be the cathedral obtained from $G$ by removing $G_{j}$ from the neighborhood of $X_{j}$ and attaching it instead as a cathedral over a barrier in an innermost chamber of $G_{i}$. The cathedrals over the barriers of innermost chambers are empty, so $G^{\prime}$ is a cathedral.

The chambers of $G$ and $G^{\prime}$ are isomorphic, so $\Phi(G)=\Phi\left(G^{\prime}\right)$, but $G^{\prime}$ has more edges. We replaced $\left|X_{j}\right| \cdot\left|V\left(G_{j}\right)\right|$ edges with $\left|X_{i}\right| \cdot\left|V\left(G_{j}\right)\right|$ edges, and also new edges were created incident to an innermost chamber over $X_{i}$. We conclude that in a $p$-extremal graph, only one maximal barrier of the foundation has a nonempty chamber over it. Also, that must be a largest barrier, since otherwise shifting to a larger one increases the number of edges, again without changing the number of perfect matchings. The claim follows by induction.

The number of edges in a spire is maximized by ordering the chambers greedily.

Lemma 4.3. Let $\left\{H_{0}, \ldots, H_{k}\right\}$ be saturated elementary graphs. Let $n_{i}=\left|V\left(H_{i}\right)\right|$, and let $s_{i}$ be the maximum size of a barrier in $H_{i}$. Among the spires having $H_{0}, \ldots, H_{k}$ as chambers, the number of edges is maximized by indexing the chambers so that $\frac{s_{0}}{n_{0}} \geq \cdots \geq \frac{s_{k}}{n_{k}}$.

Proof. For a spire $G$ generated by $H_{0}, \ldots, H_{k}$ indexed in a different order, let $i$ be an index such that $\frac{s_{i}}{n_{i}}<\frac{s_{i+1}}{n_{i+1}}$. Form a spire $G^{\prime}$ from $G$ by interchanging $H_{i}$ and $H_{i+1}$ in the ordering (always the spire after $H_{j}$ is built over a largest barrier $Y_{j}$ of $H_{j}$ ).

In $G^{\prime}$ and $G$, the edges from $Y_{i} \cup Y_{i+1}$ to other chambers are the same. Only the edges joining $V\left(H_{i}\right)$ and $V\left(H_{i+1}\right)$ change. In $G$, there are $s_{i} n_{i+1}$ such edges, and in $G^{\prime}$ there are $s_{i+1} n_{i}$ of them. By the choice of $i$, the change increases the number of edges. The number of perfect matchings remains unchanged.

Hence a $p$-extremal spire has its chambers ordered as claimed.

Note that always $\frac{s_{i}}{n_{i}} \leq \frac{1}{2}$ for a chamber $H_{i}$ in a spire $G$, since $o\left(H_{i}-X_{i}\right)=\left|X_{i}\right|$ for any barrier $X_{i}$ in $H_{i}$. We show next that the excess $c(G)$ is subadditive over the chambers.

Lemma 4.4. If $G$ is a spire generated by $H_{0}, \ldots, H_{k}$ over $Y_{0}, \ldots, Y_{k}$, with $s_{i}=\left|Y_{i}\right|$ and $n_{i}=\left|V\left(H_{i}\right)\right|$, then $c(G) \leq \sum_{i=0}^{k} c\left(H_{i}\right)$, with equality if and only if $\frac{s_{0}}{n_{0}}=\cdots=\frac{s_{k-1}}{n_{k-1}}=\frac{1}{2}$.

Proof. Let $m_{i}=\left|E\left(H_{i}\right)\right|$. Counting edges within chambers and from chambers to barriers in earlier chambers, we have $|E(G)|=\sum_{i=0}^{k} m_{i}+\sum_{0 \leq i<j \leq k} s_{i} n_{j}$. Always $s_{i} \leq \frac{1}{2} n_{i}$ and $m_{i}=\frac{1}{4} n_{i}^{2}+c\left(H_{i}\right)$. Thus

$$
\frac{n^{2}}{4}+c(H) \leq \sum_{i=1}^{k}\left[\frac{n_{i}^{2}}{4}+c\left(H_{i}\right)\right]+\sum_{0 \leq i<j \leq k} \frac{1}{2} n_{i} n_{j}=\frac{1}{4}\left[\sum_{i=0}^{k} n_{i}\right]^{2}+\sum_{i=0}^{k} c_{i} .
$$

Therefore, $c(G) \leq \sum_{i=0}^{k} c\left(H_{i}\right)$, with equality if and only if $\frac{s_{i}}{n_{i}}=\frac{1}{2}$ for $i<k$. 


\section{Extremal Chambers}

We now know how to combine chambers in the best way, so it remains to determine which chambers should be used. A chamber is a saturated elementary graph, meaning that its extendable subgraph has just one component. We will bound the size of a saturated elementary graph with $n$ vertices by bounding separately the extendable edges (those in perfect matchings) and the free edges (those in no perfect matching).

When $G$ is elementary, the maximal barriers partition $V(G)$. Since each barrier matches to vertices outside it in any perfect matching, all edges within barriers are free. Also, adding such edges does not increase the number of perfect matchings. Thus in a saturated graph, the barriers are cliques. To bound the number of free edges, the crucial fact is that in a saturated elementary graph, the only free edges are those within barriers (proved in Lemma 5.2.2.b of Lovász and Plummer [11]).

Lemma 5.1. If $G$ is a saturated elementary $n$-vertex graph with $\ell$ maximal barriers, then $G$ has at most $q\left(\begin{array}{c}\ell-1 \\ 2\end{array}\right)+\left(\begin{array}{c}r+1 \\ 2\end{array}\right)$ free edges, where $q=\left\lfloor\frac{n-\ell}{\ell-2}\right\rfloor$ and $r=n-\ell-q(\ell-2)$.

Proof. Let $x_{1}, \ldots, x_{\ell}$ be the sizes of the barriers, so $\sum_{i=1}^{\ell} x_{i}=n$. Since each barrier is a clique, there are exactly $\sum_{i=1}^{\ell}\left(\begin{array}{c}x_{i} \\ 2\end{array}\right)$ free edges. The sizes of the barriers are further restricted because deleting a barrier of size $x_{i}$ must leave $x_{i}$ odd components. Since the other barriers are cliques, deleting a barrier leaves at most $\ell-1$ components. Thus $1 \leq x_{i} \leq \ell-1$ for all $i$.

If $a \leq b$, then $\left(\begin{array}{c}a-1 \\ 2\end{array}\right)+\left(\begin{array}{c}b+1 \\ 2\end{array}\right)>\left(\begin{array}{c}a \\ 2\end{array}\right)+\left(\begin{array}{c}b \\ 2\end{array}\right)$ (shifting a vertex from an $a$-clique to a $b$-clique increases the number of edges). Subject to the constraints we have specified, the number of free edges is thus bounded by greedily choosing as many of $x_{1}, \ldots, x_{\ell}$ to equal $\ell-1$ as possible, given that at least one unit must remain for each remaining variable. Let $q$ be the number of values equal to $\ell-1$. Among the remaining values, whose total is less than $\ell-1$, all values should be 1 except for one. After allocating 1 to each of these $\ell-q$ values, a total of $r$ remains, where $0 \leq r<\ell-2$. Thus $n=q(\ell-1)+(\ell-q)+r$, which we write as $n-\ell=q(\ell-2)+r$.

The specified choice of $q$ and $r$ satisfies all the conditions, and the bound on the number of free edges is then as claimed.

We show next that the bound in Lemma 5.1 is maximized when all barriers except one are singletons, producing $\ell=1+n / 2$.

Corollary 5.2. A saturated elementary n-vertex graph has at most $\frac{n^{2}}{8}-\frac{n}{4}$ free edges.

Proof. The proof of Lemma 5.1 describes how to maximize $\sum_{i=1}^{\ell}\left(\begin{array}{c}x_{i} \\ 2\end{array}\right)$ subject to $1 \leq x_{i} \leq$ $\ell-1$. Since barriers in saturated graphs are cliques, the number of odd components left by 
deleting a barrier is at most the number of other barriers, but it must equal the size of the barrier deleted. Hence each barrier has size at most $n / 2$, which yields $\ell \leq n / 2+1$.

Thus $2 \leq \ell \leq n / 2+1$. Since $0 \leq r<\ell-2$, we have $\left(\begin{array}{c}r+1 \\ 2\end{array}\right) \leq r(\ell-1) / 2$ (with equality only when $r=0$ ). Hence

$$
q\left(\begin{array}{c}
\ell-1 \\
2
\end{array}\right)+\left(\begin{array}{c}
r+1 \\
2
\end{array}\right) \leq \frac{q(\ell-1)(\ell-2)+r(\ell-1)}{2}=\frac{(\ell-1)(n-\ell)}{2}
$$

The upper bound is maximized at $(\ell-1)=(n-1) / 2$, among integers when $\ell \in\{n / 2, n / 2+1\}$. The value there is $\frac{1}{2}\left(\frac{n}{2}-1\right)\left(\frac{n}{2}\right)$, which is the claimed bound.

Next consider the extendable edges. Deleting the edges within barriers yields a graph in which every edge is extendable. Such graphs are called 1-extendable, which motivates our name for extendable edges (the term matching-covered has also been used for 1-extendable graphs). Since the extendable edges form a 1-extendable graph, we seek a bound on the size of 1-extendable graphs with $n$ vertices. All such graphs are 2-connected, and 2-connected graphs are precisely those constructed by ear decompositions. The 1-extendable graphs have special ear decompositions that yield a bound on the number of edges, described by the "Two Ears Theorem" of Lovász.

Definition 5.3. Let $G$ be a 1-extendable graph. A graded ear decomposition of $G$ is a list $G_{0}, \ldots, G_{k}$ of 1-extendable graphs such that $G_{k}=G$, each $G-V\left(G_{i}\right)$ is matchable, and each $G_{i}$ for $i>1$ is obtained from $G_{i-1}$ by adding disjoint ears of odd length. A graded ear decomposition of $G$ is non-refinable if no other graded ear decomposition of $G$ contains it.

Theorem 5.4 (Two Ears Theorem; Lovász and Plummer [10]; see also Section 5.4 of [11]). Every 1-extendable graph has a non-refinable graded ear decomposition in which each subgraph arises by adding at most two ears to the previous one (starting with any single edge).

For example, such a decomposition of $K_{4}$ starts with any edge, adds one ear to complete a 4-cycle, and then adds both remaining edges as ears. Both ears must be added in the last step, because adding just one of them does not produce a 1-extendable graph.

Lovász and Plummer [11, page 178] remark that long graded ear decompositions are desirable, because $\Phi(G) \geq k+1$ when $G$ has a graded ear decomposition $G_{0}, \ldots, G_{k}$. We explain and use this fact in our next lemma.

Lemma 5.5. For $p \geq 2$, a 1-extendable graph $G$ with $\Phi(G)=p$ has at most $2 p-4+n$ edges.

Proof. Let $G_{0}, \ldots, G_{k}$ be an ear decomposition as guaranteed by Theorem 5.4. Since the decomposition is non-refinable, $G_{1}$ is an even cycle, so $\Phi\left(G_{1}\right)=2$. 
Let $m=|E(G)|$. The number of edges added at each step after $G_{1}$ is at most two more than the number of vertices added. Hence $m \leq n+2(k-1)$. It suffices to show that $k-1 \leq p-2$. To do this, it suffices to prove that $\Phi\left(G_{i}\right)>\Phi\left(G_{i-1}\right)$ for $i \geq 2$.

Every added ear in a graded ear decomposition has odd length and hence an even number of internal vertices. These can be matched along the ear. Since $G_{i}$ arises from $G_{i-1}$ by adding one ear of odd length or two disjoint ears of odd length, every perfect matching in $G_{i-1}$ extends to a perfect matching in $G_{i}$. In addition, since $G_{i}$ is also required to be 1extendable, it has a perfect matching using an initial edge of an added ear; such a matching is not counted by $\Phi\left(G_{i-1}\right)$.

Theorem 5.6. For $p \geq 2$, an elementary graph with $n$ vertices and exactly $p$ perfect matchings has at most $\frac{n^{2}}{8}+\frac{3 n}{4}+2 p-4$ edges.

Proof. Add the maximum number of extendable edges from Lemma 5.5 to the maximum number of free edges from Corollary 5.2.

Since the coefficient on the quadratic term in this edge bound is $\frac{1}{8}$, while the leading coefficient for $p$-extremal graphs will be $\frac{1}{4}$, large extremal graphs will not be elementary. This enables us to limit the search for extremal elementary graphs.

Corollary 5.7. Fix $p \geq 2$. If $G$ is an elementary graph with $n$ vertices, $p$ perfect matchings, and $\frac{n^{2}}{4}+c_{p}$ edges, then $n^{2}-6 n-16 p+8 c_{p}+32 \leq 0$. Thus $n \leq 3+\sqrt{16 p-8 c_{p}-23}$.

Recall that $n_{p}=\min \left\{n: f(n, p)=\frac{n^{2}}{4}+c_{p}\right\}$. We can bound this threshold using the fact that all the chambers in a spire are elementary graphs.

Corollary 5.8. For $p \geq 2$, let $N_{p}$ be the largest even number bounded by $3+\sqrt{16 p-8 c_{p}-23}$. Every elementary graph in $\mathcal{F}_{p}$ has at most $N_{p}$ vertices, and $n_{p} \leq \max \left\{\sum_{i=0}^{k} N_{p_{i}}: \prod_{i} p_{i}=p\right\}$.

Proof. By Corollary 5.7, all elementary graphs with $n$ vertices and $\frac{n^{2}}{4}+c_{p}$ edges have at most $3+\sqrt{16 p-8 c_{p}-23}$ vertices, and the number of vertices must be even.

Let $G \in \mathcal{F}_{p}$ be a spire generated by $H_{0}, \ldots, H_{k}$. Set $p_{i}=\Phi\left(H_{i}\right)$. We have observed that $p=\prod_{i=0}^{k} p_{i}$. Since each $H_{i}$ is elementary, it has at most $N_{p_{0}}$ vertices, so $G$ has at most $\sum_{i=1}^{k} N_{p_{i}}$ vertices. Taking the maximum over all factorizations bounds $n_{p}$.

The lower bound $c_{p} \geq-(p-1)(p-2)$ given by Dudek and Schmitt [5] implies $N_{p} \in O(p)$. The construction in Theorem 2.3 shows that $c_{p}$ is nonnegative. Together with Corollary 5.8, this yields $N_{p} \in O(\sqrt{p})$. With $N_{q}$ known for $q<p$, this reduces the determination of the exact value of $c_{p}$ for a given $p$ to a search over a finite set of graphs.

We close this section by summarizing the results of this and the previous section. The outcome is a systematic approach to classifying all graphs in $\mathcal{F}_{p}$. 
Theorem 5.9. For an n-vertex graph $G$ in $\mathcal{F}_{p}$,

1. $G$ is a spire with chambers $H_{0}, \ldots, H_{k}$ built over barriers $Y_{0}, \ldots, Y_{k}$.

2. Each $Y_{i}$ is a barrier of maximum size in $H_{i}$.

3. If $0 \leq i<j \leq k$, then $\frac{\left|Y_{i}\right|}{\left|V\left(H_{i}\right)\right|} \leq \frac{\left|Y_{j}\right|}{\left|V\left(H_{j}\right)\right|}$.

4. Letting $p_{i}=\Phi\left(H_{i}\right)$, there are at most $N_{p_{i}}$ vertices in $H_{i}$, and $c\left(H_{i}\right) \leq c_{p_{i}}$.

5. $\Phi(G)=p=\prod_{i=0}^{k} p_{i}$ and $c(G)=c_{p} \leq \sum_{i=1}^{k} c\left(H_{i}\right)$.

6. If $p_{i}=1$, then $H_{i} \cong K_{2}$.

\section{Graphs with an Odd Number of Vertices}

Since graphs with an odd number of vertices do not have perfect matchings, we generalize $f(n, p)$ to odd $n$ using near-perfect matchings. In this section, $n$ is odd.

Definition 6.1. An near-perfect matching in a graph is a matching that covers all but one vertex. Let $\tilde{\Phi}(G)$ denote the number of near-perfect matchings in $G$. Let $\tilde{f}(n, p)$ denote the maximum number of edges in an $n$-vertex graph with $p$ near-perfect matchings.

The computation of $\tilde{f}(n, p)$ almost reduces to the computation of $f(n, p)$.

Theorem 6.2. If $n$ is odd and larger than $n_{p}$, then

$$
\tilde{f}(n, p)=f(n-1, p)=\frac{(n-1)^{2}}{4}+c_{p} .
$$

Proof. Since $n>n_{p}$, we may choose $G \in \mathcal{F}_{p}$ with $n-1$ vertices. Adding an isolated vertex to $G$ produces a graph with $p$ near-perfect matchings and $f(n-1, p)$ edges. Thus $f(n-1, p) \leq \tilde{f}(n, p)$.

Let $H$ be an $n$-vertex graph having $\tilde{f}(n, p)$ edges and $p$ near-perfect matchings. Adding a new vertex adjacent to every vertex in $H$ produces a graph $H^{\prime}$ having $p$ perfect matchings and $\tilde{f}(n, p)+n$ edges (there is a one-to-one correspondence between near-perfect matchings in $H$ and perfect matchings in $\left.H^{\prime}\right)$.

Thus $\tilde{f}(n, p)=\left|E\left(H^{\prime}\right)\right|-n \leq f(n+1, p)-n$. By Theorem 1.2, $n>n_{p}$ implies $f(n+1, p)=$ $f(n-1, p)+n$. We conclude that $\tilde{f}(n, p) \leq f(n-1, p)$, so equality holds.

Not only is the numerical value of $\tilde{f}(n, p)$ determined by the even case, but also the extremal graphs correspond to extremal graphs in the even case, using the bijection in the proof of Theorem 6.2. 
Definition 6.3. Let $\tilde{\mathcal{F}}_{p}$ be the set of graphs $G$ having $\frac{(|V(G)|-1)^{2}}{4}+c_{p}$ edges and exactly $p$ near-perfect matchings.

Corollary 6.4. For each graph $H \in \tilde{\mathcal{F}}_{p}$, there is a graph $G \in \mathcal{F}_{p}$ and a vertex $u \in V(G)$ such that $u$ is adjacent to $V(G)-\{u\}$ and $H \cong G-u$.

Not every graph in $\mathcal{F}_{p}$ has a dominating vertex, so there are $n$-vertex graphs in $\mathcal{F}_{p}$ that do not arise in this simple way from $(n-1)$-vertex graphs in $\tilde{\mathcal{F}}_{p}$. The graph $\overline{3 K_{2}}$ has eight perfect matchings (each of the 12 edges appears in two perfect matchings, and each perfect matching has three edges). With $n=6$, we have $n^{2} / 4+3$ edges. We will see that $c_{8}=3$, so $\overline{3 K_{2}} \in \mathcal{F}_{p}$, but the graph has no dominating vertex. On the other hand, when $n>n_{p}$, Hetyei-extension of an $n$-vertex graph in $\mathcal{F}_{p}$ yields a graph in $\mathcal{F}_{p}$ with $n+2$ vertices that does have a dominating vertex.

\section{Constructive Lower Bounds}

In this section, we refine the binary expansion construction $B(p)$ of Theorem 2.3 to give improved lower bounds for $c_{p}$. Because the barrier is large in $B(p)$, it can be used to increase the excess while multiplying the number of perfect matchings. Recall that $w(m)$ is the number of $1 \mathrm{~s}$ in the binary expansion of $m$.

Proposition 7.1. If $p_{1}$ and $p_{2}$ are integers with $p_{1}, p_{2} \geq 2$, then $c_{p_{1} p_{2}} \geq c_{p_{1}}+w\left(p_{2}-1\right)$.

Proof. Let $G$ be a $n$-vertex graph having $\frac{n^{2}}{4}+c_{p_{1}}$ edges and exactly $p_{1}$ perfect matchings. Let $H=B\left(p_{2}\right)$, in which the clique is a barrier containing exactly half of the vertices. Let $G^{\prime}$ be the saturated graph formed by making $G$ a tower above this barrier in $H$.

By Lemma 4.4, $c\left(G^{\prime}\right)=c(G)+c(H)=c_{1}+w(p-1)$. By Lemma 3.2, $G^{\prime}$ has $p_{1} \cdot p_{2}$ distinct perfect matchings. Therefore, $c_{p_{1} p_{2}} \geq c_{p_{1}}+w\left(p_{2}-1\right)$.

Corollary 7.2. If properly divides $p^{\prime}$, then $c_{p^{\prime}}>c_{p}$.

The binary expansion construction yields $c_{p} \geq \log _{2} p$ when $p$ is a power of 2 . However, when $p-1$ is a power of 2 , it yields only $c_{p} \geq 1$. To combat this deficiency, we develop further lower bounds using graphs where $|E(G)|$ and $\Phi(G)$ are easy to compute. These constructions properly contain the Hetyei graphs, so the excess is positive. Unfortunately, not every $p$ can be realized as $\Phi(G)$ using these constructions.

Definition 7.3. A Hetyei list is a nondecreasing list $d_{1}, \ldots, d_{k}$ of positive integers such that $d_{i} \geq i$ for all $i$ and $d_{k}=k$. The nested-degree graph generated by a Hetyei list $\left(d_{1}, \ldots, d_{k}\right)$, denoted $\operatorname{Deg}\left(d_{1}, \ldots, d_{k}\right)$, is the supergraph of the Hetyei graph of order $2 k$ in which the edge $\ell_{i} r_{j}$ exists if and only if $i \leq d_{j}$. 
Theorem 7.4. If $G=\operatorname{Deg}\left(d_{1}, \ldots, d_{k}\right)$ for a Hetyei list $d_{1}, \ldots, d_{k}$, then $G$ has a barrier of size $k$ and $\Phi(G)=\prod_{i=1}^{k}\left(d_{i}+1-i\right)$,

Proof. Since $\left\{r_{1}, \ldots, r_{k}\right\}$ is an independent set, every perfect matching pairs its vertices with $\left\{\ell_{1}, \ldots, \ell_{k}\right\}$. Also, $\left\{\ell_{1}, \ldots, \ell_{k}\right\}$ is a barrier of size $k$ in $G$.

To compute $\Phi(G)$, choose edges to cover vertices in the order $r_{1}, \ldots, r_{k}$. When covering $r_{i}$, there are $i-1$ previously matched vertices in $\left\{\ell_{1}, \ldots, \ell_{k}\right\}$. Since $\bigcup_{j=1}^{i-1} N\left(r_{i}\right) \subseteq N\left(r_{i}\right)$, there are $d_{i}-i+1$ choices for the edge to cover $r_{i}$. Since $d_{i} \geq i$ for all $i$, the process completes a perfect matching in $\prod_{i=1}^{k}\left(d_{i}+1-i\right)$ ways.

When a graph $G$ has a barrier $B$ with half its vertices, the edges in perfect matchings form a bipartite graph with partite sets $B$ and $V(G)-B$, and $G-B$ has no edges. Ostrand [15] proved that if a bipartite graph $G$ has a perfect matching, and $d_{1}, \ldots, d_{k}$ is the nondecreasing list of degrees of the vertices in one partite set, then $\Phi(G) \geq \prod_{i=1}^{k} \max \left\{1, d_{i}-i+1\right\}$ (Hwang [9] gave a simple proof). When the list $d$ is a Hetyei list, the corresponding nested-degree graphs achieve equality in the lower bound.

Example 7.5. If $G=\operatorname{Deg}(k, \ldots, k)$, then $\Phi(G)=k$ ! and $c(G)=\left(\begin{array}{l}k \\ 2\end{array}\right)$. By Stirling's approximation, $c_{p} \geq \Omega\left(\left(\frac{\ln p}{\ln \ln p}\right)^{2}\right)$ when $p=k !$.

Definition 7.6. Let $\left(d_{1}, \ldots, d_{k}\right)$ be a Hetyei list and $\left\{e_{1}, \ldots, e_{m}\right\}$ be a set of disjoint pairs in $\{1, \ldots, k\}$. The resulting generalized nested-degree graph, denoted Gen $\left(d_{1}, \ldots, d_{k} ; e_{1}, \ldots, e_{m}\right)$, consists of the nested-degree graph $\operatorname{Deg}\left(d_{1}, \ldots, d_{k}\right)$ plus each edge $r_{i} r_{j}$ such that $\{i, j\}=e_{t}$ for some $t$.

The double factorial of an integer $n$, denoted $n !$ !, is the product of the integers in $\{1, \ldots, n\}$ with the same parity as $n$. As an empty product, by convention $(-1)$ !! equals 1 .

Theorem 7.7. For a set $\left\{e_{1}, \ldots, e_{m}\right\}$ of disjoint pairs in $\{1, \ldots, k\}$, let $\mathcal{P}$ denote the family of all subsets of $\left\{r_{i} r_{j}:\{i, j\} \in\left\{e_{1}, \ldots, e_{m}\right\}\right\}$. If $G=\operatorname{Gen}\left(d_{1}, \ldots, d_{k} ; e_{1}, \ldots, e_{m}\right)$, then

$$
\Phi(G)=\sum_{M \in \mathcal{P}}(2|M|-1) ! ! \prod_{r_{i} \notin V(M)}\left(d_{i}-\left|\left\{j<i: r_{j} \notin V(M)\right\}\right|\right) .
$$

Also, if $m \geq 1$, then $G$ has no barrier of size $k$.

Proof. Every perfect matching in $G$ contains some subset $M$ of $\left\{r_{i} r_{j}:\{i, j\} \in\left\{e_{1}, \ldots, e_{m}\right\}\right\}$. To complete a matching, cover the remaining vertices in $\left\{r_{1}, \ldots, r_{k}\right\}$ in increasing order of subscripts by selecting neighbors in $\left\{\ell_{1}, \ldots, \ell_{k}\right\}$. The number of ways to do this is $\prod_{r_{i} \notin V(M)}\left(d_{i}-\left|\left\{r_{j} \notin V(M): j<i\right\}\right|\right)$, as in the proof of Theorem 7.4. Finally, the $2|M|$ remaining unmatched vertices form a clique and can be matched in $(2|M|-1)$ !! ways. 
Theorem 7.4 is the special case of Theorem 7.7 for $m=0$. When $m$ is small, there are not many subsets of $\left\{e_{1}, \ldots, e_{m}\right\}$, and computing $\Phi(G)$ is feasible.

Example 7.8. When $m=\left(\begin{array}{c}k \\ 2\end{array}\right)$ and $d_{i}=k$ for all $i$, the generalized nested-degree graph is $K_{2 k}$, with $(2 k-1)$ !! perfect matchings. Thus $c_{(2 k-1) ! !} \geq k^{2}-k$. This yields the lower bound $c_{p} \geq \Omega\left(\left(\frac{\ln p}{\ln \ln p}\right)^{2}\right)$ when $p=(2 m-1)$ !! for some $m$.

Examples 7.5 and 7.8 provide our best asymptotic lower bounds but apply only for special values. The generalized nested-degree construction is our most efficient method for finding lower bounds when $k$ and $m$ are small In Section 9, we discuss the results of computer search over small cases of these constructions to find explicit lower bounds on $c_{p}$ when $p$ is small.

\section{A Conjectured Upper Bound}

Dudek and Schmitt conjectured that the complete graph $K_{2 t}$ is $p$-extremal for $p=(2 t-1) !$ !, giving $c_{p}=t^{2}-t$. We generalize this to conjecture an upper bound for all $p$. First, a lemma provides motivation. In light of the proof, we call it the "Star-Removal Lemma".

Lemma 8.1. If $p, k, t \in \mathbf{N}$ satisfy $k \leq 2 t$ and $p=k(2 t-1)$ !!, then $c_{p} \geq t^{2}-t+k-1$.

Proof. Let $G$ be the graph obtained from $K_{2 t+2}$ by removing $2 t+1-k$ edges with a common endpoint $x$. The vertex $x$ has $k$ neighbors; after choosing one, the rest of the graph is isomorphic to $K_{2 t}$. Thus $\Phi(G)=k(2 t-1)$ !!. The number of edges in $G$ is $\left(\begin{array}{c}2 t+2 \\ 2\end{array}\right)-(2 t+1-k)$, which equals $\frac{(2 t+2)^{2}}{4}+t^{2}-t+k-1$. Hence $c_{p} \geq t^{2}-t+k-1$.

To reduce the number of perfect matchings from $(2 t+1)$ !! to $k(2 t-1)$ !! , only $2 t-1-k$ edges were removed; with each edge deleted, $(2 t-1)$ !! perfect matchings were lost. This seems to be the most edge-efficient way to remove perfect matchings, which suggests a conjecture.

Conjecture 8.2. For $p \in \mathbf{N}$, if integers $k$ and $t$ are defined uniquely by $k(2 t-1) ! ! \leq p<$ $(k+1)(2 t-1) !$ ! with $k \leq 2 t$, then $c_{p} \leq C_{p}$, where $C_{p}=t^{2}-t+k-1$.

The conjecture matches the lower bound in Lemma 8.1 when $p=k(2 t-1)$ !!. It also matches the value of $c_{p}$ for $p \leq 6$ as computed in [5]. In Section 9, we verify that $C_{p}$ also equals $c_{p}$ for $7 \leq p \leq 10$, and we give empirical evidence that the bound holds for all $p$.

\section{Exact Values for Small $p$}

To confirm the values of $c_{p}$ for $p \leq 6$, we used McKay's geng program [12, 13] to generate all graphs on 10 vertices. We checked that none of these graphs have exactly $p$ perfect matchings 
while achieving larger excess. This yields a proof, since $N_{p} \leq 10$ for $p \leq 6$ and the smallest graph in $\mathcal{F}_{p}$ has at most $N_{p}$ vertices.

For $p \leq 10$, we have $N_{p} \leq 12$. Generating all graphs on 12 vertices presently is infeasible for us; instead, we use the following lemma.

Lemma 9.1 (Dudek-Schmitt [5, Lemma 2.4]). If $p \geq 2$, then $c_{p} \leq 1+\max \left\{c_{q}: q<p\right\}$,

If we know all previous values of $c_{p}$, and we construct an $n$-vertex graph $G$ with $\Phi(G)=p$ and $|E(G)|=\frac{n^{2}}{4}+C$, where $C=\max \left\{c_{q}: q<p\right\}$, then we only need to check graphs with $\frac{n^{2}}{4}+C+1$ edges to see whether one has exactly $p$ perfect matchings. Thus our proof of the next theorem is by computer search. It yields the values in Table 1.

\begin{tabular}{|r|c|c|c|c|c|c|c|c|c|c|}
\hline$p$ & 1 & 2 & 3 & 4 & 5 & 6 & 7 & 8 & 9 & 10 \\
\hline$c_{p}$ & 0 & 1 & 2 & 2 & 2 & 3 & 3 & 3 & 4 & 4 \\
$n_{p}$ & 2 & 4 & 4 & 6 & 6 & 6 & 6 & 6 & 6 & 6 \\
$N_{p}$ & & 4 & 6 & 8 & 8 & 10 & 10 & 12 & 12 & 12 \\
\hline & \multicolumn{3}{|c|}{$[5]$} \\
\hline
\end{tabular}

Table 1: Excess $c_{p}$ (at $n_{p}$ ), bound $N_{p}$ on extremal chambers.

Theorem 9.2. $c_{7}=3, c_{8}=3, c_{9}=4$, and $c_{10}=4$.

Proof. Explicit constructions in Fig. 5 give the lower bounds; we will subsequently describe how these constructions arise.

For the first two upper bounds, Lemma 9.1 yields (a) $c_{7} \leq 4$ and (b) if $c_{7}=3$, then $c_{8} \leq 4$. Thus to show $c_{7}=c_{8}=3$ it suffices to examine graphs with 12 vertices and $\frac{12^{2}}{4}+4$ edges. Using geng, we generated these and found none with exactly seven or eight perfect matchings, so $c_{7}=c_{8}=3$.

By Lemma 9.1, $c_{9} \leq 4$, and then similarly $c_{10} \leq 5$. To test equality, it suffices to study graphs with 12 vertices and $\frac{12^{2}}{4}+5$ edges. Using geng, we enumerated these and found no graph with exactly ten perfect matchings, so $c_{10} \leq 4$.

For small $p$, the chambers in the $p$-extremal graphs are instances of the general constructions we have provided in earlier sections. Below we characterize all $p$-extremal graphs for $p \leq 10$. Fig. 5 shows the smallest instances of the classes of graphs in these characterizations. The edge-colorings indicate the decomposition into chambers. Blue edges are extendable; when the subgraph of blue edges is connected, the graph is elementary. Red edges indicate the maximal barriers in chambers. Faint edges join these barriers to the spires over them when the graph is not elementary, in which case the factorization of $p$ should be apparent; recall that $\Phi(G)$ is the product of $\Phi\left(H_{i}\right)$ when the chambers of $G$ are $H_{0}, \ldots, H_{k}$. 

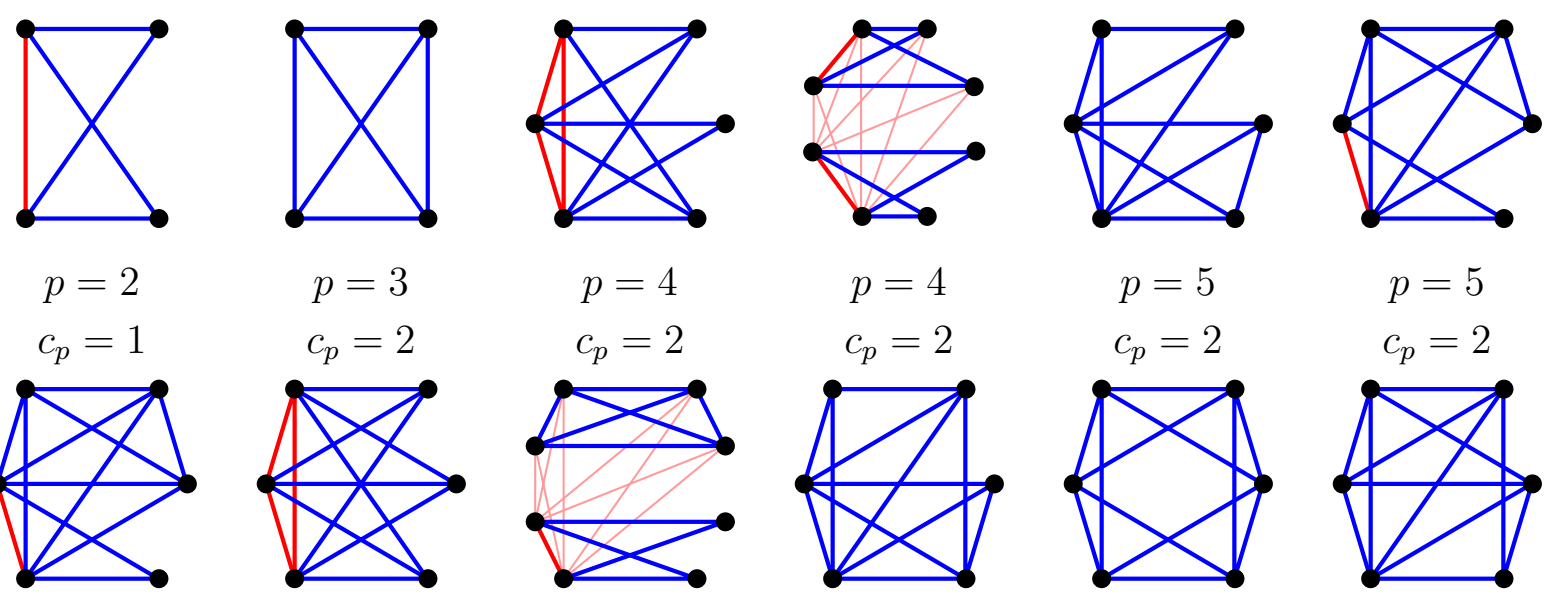

$p=3$

$p=4$

$p=4$

$p=5$

$p=5$

$c_{p}=2$

$$
c_{p}=2
$$

$c_{p}=2$

$c_{p}=2$
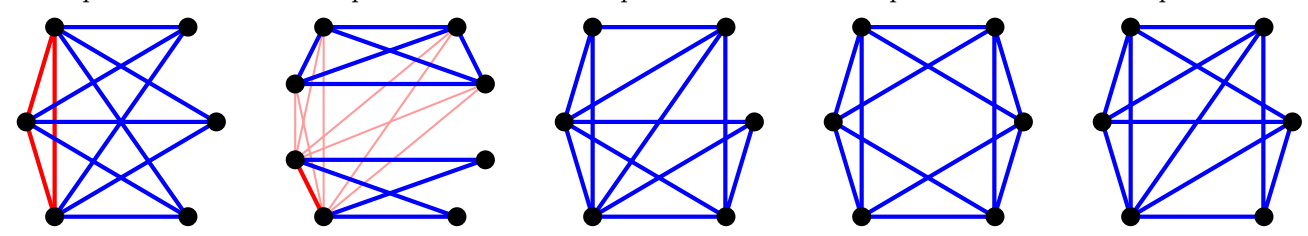

$p=6$

$p=6$

$p=6$

$p=7$

$p=8$

$p=8$

$c_{p}=3$

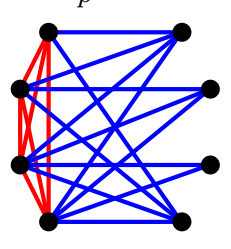

$c_{p}=3$

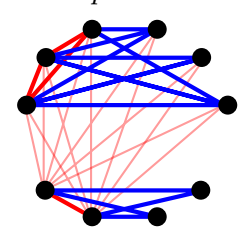

$c_{p}=3$

$c_{p}=3$

$c_{p}=3$

$c_{p}=3$
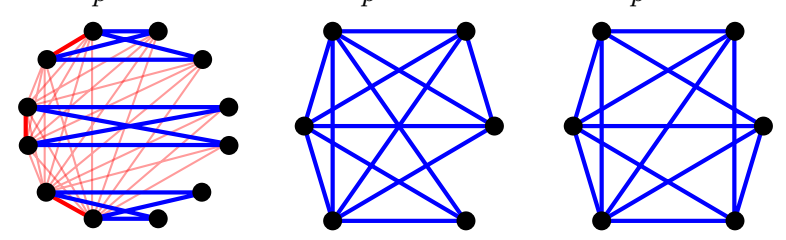

$p=8$

$p=8$

$p=8$

$p=9$

$p=10$

$c_{p}=3$

$c_{p}=3$

$c_{p}=3$

$c_{p}=4$

$c_{p}=4$

Figure 5: The smallest $p$-extremal configurations, for $2 \leq p \leq 10$

Hetyei characterized the 1-extremal graphs. Dudek and Schmitt determined $c_{p}$ for $p \leq 6$ but provided proof only for $p \leq 4$ and characterized the extremal graphs only for $p \leq 3$. We restate these characterizations in the language of the Cathedral Theorem. The "top" of a spire is the chamber last in the list of chambers describing the cathedral decomposition; it is at the other end from the foundation. When $G$ is edge-transitive, $G^{-}$denotes the graph obtained by deleting one edge from $G$.

Theorem 9.3 (Hetyei). For even $n$ with $n \geq 2$, the unique 1-extremal graph has $\frac{n^{2}}{4}$ edges and is a spire whose chambers all equal $K_{2}$.

Theorem 9.4 ([5]). For even $n$ with $n \geq 4$, the 2 -extremal graphs have $\frac{n^{2}}{4}+1$ edges and are spires whose chambers are $(n-4) / 2$ copies of $K_{2}$ and one copy of $K_{4}^{-}$, taken in any order.

Theorem 9.5 ([5]). For even $n$ with $n \geq 4$, the unique 3 -extremal graph has $\frac{n^{2}}{4}+2$ edges and is a spire whose chambers are $(n-4) / 2$ copies of $K_{2}$ and one copy of $K_{4}$ at the top.

Note that $K_{2}$ and $K_{4}^{-}$have a barrier containing half of the vertices, while $K_{4}$ does not; hence the chambers for $p=2$ appear in any order, while $K_{4}$ is at the top when $p=3$. 
The characterizations of the $p$-extremal graphs for $4 \leq p \leq 10$ all use the same method and involve the computer search used to prove Theorem 9.2. Instead of repeating the observations for each proof, we outline them here and just state the resulting characterizations.

Outline of Characterization Proofs. A $p$-extremal graph $G$ is a spire of chambers $H_{0}, \ldots, H_{k}$ (Lemma 4.2), and $c(G) \leq \sum_{i} c\left(H_{i}\right)$ (Lemma 4.4). The number of perfect matchings in $G$ equals $\prod_{i} \Phi\left(H_{i}\right)$ (Lemma 3.2). Hence to know the $p$-extremal graphs it suffices to know the $p_{j}$-extremal chambers for all $p_{j}$ that are factors of $p$ and compare the numbers of edges in the spires corresponding to factorizations of $p$.

The chambers in spires are elementary graphs. Every $p$-extremal elementary graph has at most $N_{p}$ vertices, where $N_{p}$ is the largest even number bounded by $3+\sqrt{16 p-8 c_{p}-23}$ (Corollary 5.8). A $p$-extremal elementary graph with fewer than $N_{p}$ vertices extends to a $p$-extremal graph with $N_{p}$ vertices by Hetyei-extension (repeatedly adding $K_{2}$ as a chamber at the beginning of the spire), so the $p$-extremal chambers are found within the graphs on $N_{p}$ vertices. The $q$-extremal chambers for $q<p$ are already known from previous searches.

When searching graphs with $N_{p}$ vertices for $p$-extremal chambers, we limit the search to specific numbers of edges. A $p$-extremal graph with $N_{p}$ vertices has $\frac{1}{4} N_{p}^{2}+c_{p}$ edges. By Lemma 9.1, $c_{p} \leq C+1$, where $C=\max _{q<p} c_{q}$. Hence we begin by searching graphs with $N_{p}$ vertices and excess $C+1$, looking for those having exactly $p$ perfect matchings. The search moves to excess $C$ if none are found with excess $C+1$. In the results for $p \leq 10$, graphs with $N_{p}$ vertices and $p$ perfect matchings were always found having excess $C$ or $C+1$, so there was no need to search further.

At this point the $q$-extremal chambers are known for all factors $q$ of $p$, and hence the complete description of $p$-extremal graphs can be given. The chambers in a $p$-extremal spire are $q_{i}$-extremal elementary graphs, where $\prod q_{i}=p$. However, a spire with $q_{i}$-extremal chambers may have too few edges to be $\prod q_{i}$-extremal (for example, the spire with chambers $K_{4}$ and $K_{4}$ has nine perfect matchings but is not 9-extremal).

The order of chambers in a spire does not affect the number of perfect matchings, but it does affect the number of edges. To have the most edges, the chambers must be listed in decreasing order of the fractions of their vertices occupied by their largest barrier (Lemma 4.3). Spires for which these fractions are equal (such as $K_{2}$ and $K_{4}^{-}$having barriers with half their vertices) may be listed in any order.

See Fig. 5 for the smallest instances of the classes of graphs in these characterizations.

Theorem 9.6. For even $n$ with $n \geq 6$, the 4 -extremal graphs have $\frac{n^{2}}{4}+2$ edges and are spires whose chambers are

a) $\frac{n-6}{2}$ copies of $K_{2}$ and one copy of $B(4)$ in any order, or

b) $\frac{n-8}{2}$ copies of $K_{2}$ and two copies of $K_{4}^{-}$in any order. 
Theorem 9.7. For even $n$ with $n \geq 6$, the 5-extremal graphs have $\frac{n^{2}}{4}+2$ edges and are spires whose chambers are $\frac{n-6}{2}$ copies of $K_{2}$ plus one 6-vertex graph at the top that is $\operatorname{Gen}(2,2,3 ;\{1,2\})$ or $\operatorname{Gen}(2,3,3 ;\{2,3\}))-\ell_{2} r_{2}$.

Theorem 9.8. For even $n$ with $n \geq 6$, the 6 -extremal graphs have $\frac{n^{2}}{4}+3$ edges and are spires whose chambers are

a) $\frac{n-6}{2}$ copies of $K_{2}$ and one copy of $\operatorname{Gen}(2,3,3 ;\{2,3\})$ at the top,

b) $\frac{n-6}{2}$ copies of $K_{2}$ and one copy of $\operatorname{Deg}(3,3,3)$ in any order, or

c) $\frac{n-8}{2}$ copies of $K_{2}$ and one copy of $K_{4}^{-}$in any order, plus one copy of $K_{4}$ at the top.

Theorem 9.9. For even $n$ with $n \geq 6$, the unique 7 -extremal graph has $\frac{n^{2}}{4}+3$ edges and is a spire whose chambers are $\frac{n-6}{2}$ copies of $K_{2}$ and one $\operatorname{Gen}(2,2,3 ;\{1,2\},\{1,3\})$ at the top.

Theorem 9.10. For even $n$ with $n \geq 6$, the 8-extremal graphs have $\frac{n^{2}}{4}+3$ edges and are spires whose chambers are a) $\frac{n-6}{2}$ copies of $K_{2}$, plus one copy of $\overline{3 K_{2}}$ at the top,

b) $\frac{n-6}{2}$ copies of $K_{2}$, plus one copy of $\operatorname{Gen}(1,3,3 ;\{1,2\},\{1,3\},\{2,3\})$ at the top,

c) $\frac{n-8}{2}$ copies of $K_{2}$ and one copy of $B(8)$ in any order,

d) $\frac{n-10}{2}$ copies of $K_{2}$, one copy of $K_{4}^{-}$, and one copy of $B(8)$ in any order, or

e) $\frac{n-12}{2}$ copies of $K_{2}$ and three copies of $K_{4}^{-}$in any order.

Theorem 9.11. For even $n$ with $n \geq 6$, the unique 9 -extremal graph has $\frac{n^{2}}{4}+4$ edges and is a spire whose chambers are $\frac{n-6}{2}$ copies of $K_{2}$ and one $\operatorname{Gen}(3,3,3 ;\{2,3\})$ at the top.

Theorem 9.12. For even $n$ with $n \geq 6$, the unique 10-extremal graph has $\frac{n^{2}}{4}+4$ edges and is a spire whose chambers are $\frac{n-6}{2}$ copies of $K_{2}$ and one $\operatorname{Gen}(2,3,3 ;\{1,2\},\{1,3\})$ at the top.

Moving beyond $p=11$, note that $N_{11}=14$. Unfortunately, the number of graphs with 14 vertices and suitable number of edges is beyond the capacity of our computer resources to determine $c_{11}$ by this method.

In Figure 6, we present the lower bounds on $c_{p}$ found by searching all graphs of order 10 to find chambers and forming spires from these chambers and chambers arising from the generalized nested degree construction on 12,14 , and 16 vertices with $k \in\{5,6,7,8\}$ and $m \in\{4,3,2,1\}$. The upper line is the conjectured upper bound $C_{p}$ from Conjecture 8.2, defined as $t^{2}-t+k-1$, where $t$ and $k$ are determined by $k(2 t-1) ! ! \leq p<(k+1)(2 t-1) !$ ! with $k \leq 2 t$. As the plot shows, we have found no construction that violates the upper bound, and sometimes it equals the excess of the best construction found so far.

Using the structural theorems in this paper, a subsequent paper [17] develops a method for searching all 1-extendable graphs with at most $p$ perfect matchings and then adding edges to find dense elementary graphs. The 1-extendable graphs are built using graded ear 


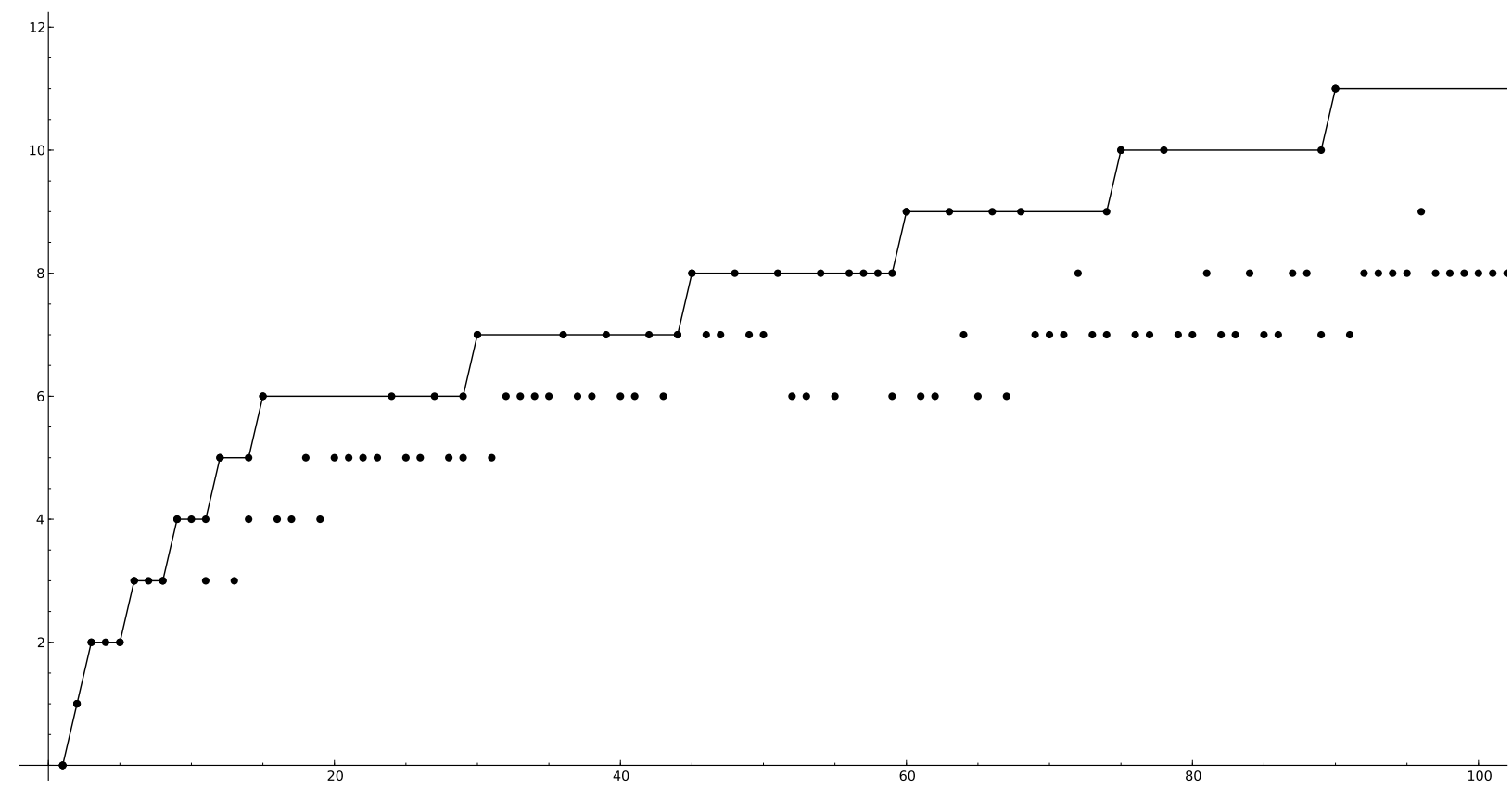

(a) $1 \leq p \leq 100$

Figure 6: Lower bounds on $c_{p}$ and conjectured upper bound $C_{p}$.

decompositions. Since all 1-extendable graphs are explored, adding free edges generates all spires with at most $p$ perfect matchings, thus determining $c_{p}$. This method produces the values of $c_{p}$ for $p \leq 27$ and provides the elementary graphs that attain the maximum excess. The resulting values of $c_{p}$ appear in Table 2 . The conjectured upper bound $C_{p}$ is not always sharp; more surprising is that $c_{p}$ is not monotone.

\begin{tabular}{|r|c|c|c|c|c|c|c|c|c|c|c|c|c|c|c|c|c|}
\hline$p$ & 11 & 12 & 13 & 14 & 15 & 16 & 17 & 18 & 19 & 20 & 21 & 22 & 23 & 24 & 25 & 26 & 27 \\
\hline$N_{p}$ & 14 & 14 & 14 & 16 & 16 & 16 & 16 & 18 & 18 & 18 & 18 & 20 & 20 & 20 & 20 & 20 & 22 \\
$C_{p}$ & 4 & 5 & 5 & 5 & 6 & 6 & 6 & 6 & 6 & 6 & 6 & 6 & 6 & 6 & 6 & 6 & 6 \\
\hline$c_{p}$ & 3 & 5 & 3 & 4 & 6 & 4 & 4 & 5 & 4 & 5 & 5 & 5 & 5 & 6 & 5 & 5 & 6 \\
$n_{p}$ & 8 & 6 & 8 & 8 & 6 & 8 & 8 & 8 & 8 & 8 & 8 & 8 & 8 & 8 & 8 & 8 & 8 \\
\hline
\end{tabular}

Table 2: New values of $N_{p}, C_{p}, c_{p}$, and $n_{p}$.

The method we used to determine the graphs in $\mathcal{F}_{p}$ for $p \leq 10$ is feasible only for small $p$. Several natural questions arise from these computational results. The data suggest that there is always at least one $p$-extremal graph whose subgraph of extendable edges is connected. If this is true, then it could help to guide searches, since when $n \geq n_{p}$ some $p$-extremal graph would have a chamber other than $K_{2}$ only at the top (this must happen when $p$ is prime). 
Conjecture 9.13. For $p \in \mathbf{N}$, there exist a p-extremal graph that is an elementary graph.

For $p$-extremal spires that consist of copies of $K_{2}$ and one $p$-extremal chamber, the value of $c_{p}$ may be small. The examples we have of $c_{p}<c_{p-1}$ occur when $p$ is a power of a prime, at $p \in\{11,13,16,19,25\}$. With greater variety of factorizations available, there are more ways to form spires with exactly $p$ perfect matchings and hence more ways for $c_{p}$ to be large. The data suggest the following.

Conjecture 9.14. For $p \in \mathbf{N}$, always $c_{p} \geq \max \left\{\sum c_{i}\left\{p_{i}\right\}: \prod p_{i}=p\right\}$.

A different conjecture is suggested by consider the values of $c_{q}$ after $c_{p}$. Let $m_{p}=$ $\min \left\{c_{q}: q \geq p\right\}$; how does this sequence behave? We know only that $m_{p} \geq 1$, by Theorem 2.3. However, if there are finitely many Fermat primes (primes of the form $2^{k}+1$, see [8]), then $m_{p} \geq 2$ for sufficiently large $p$, by Corollary 7.2. This is too difficult a task for such a small payoff, especially since a much stronger statement seems likely.

Conjecture 9.15. $\lim _{p \rightarrow \infty} m_{p}=\infty$.

Figure 6 provides strong support for this conjecture. If it holds, then what is the asymptotic behavior of $m_{p}$ ? Is it $\Omega\left(\left(\frac{\ln p}{\ln \ln p}\right)^{2}\right)$, matching the conjectured upper bound? Or, is it smaller, such as $\Omega\left(\log _{2} p\right)$, the current lower bound when $p$ is a power of 2 ?

Finally, it would be interesting to characterize $\mathcal{F}_{p}$ or at least compute $c_{p}$ for some infinite family of values of $p$.

\section{Acknowledgements}

The authors thank Garth Isaak for suggesting the extension to odd $n$.

\section{References}

[1] N. Alon and S. Friedland, The maximum number of perfect matchings in graphs with a given degree sequence. Electron. J. Combin. 15 (2008), Paper N13, 2 pp.

[2] L. M. Brègman, Some properties of nonnegative matrices and their permanents, Soviet Math. Dokl. 14 (1973), 945-949.

[3] B. Cuckler and J. Kahn, Entropy bounds for perfect matchings and Hamiltonian cycles, to appear.

[4] J. Cutler and A. J. Radcliffe, An entropy proof of the Kahn-Lovász Theorem. Electron. J. Combin. 18(1) (2011), Paper P10, 9 pp. 
[5] A. Dudek and J. Schmitt, On the size and structure of graphs with a constant number of 1-factors (2010), submitted.

[6] S. Friedland, An upper bound for the number of perfect matchings in graphs, arXiv: 0803.0864v1, 6 March 2008.

[7] D. Gross, N. Kahl, and J. T. Saccoman, Graphs with the maximum or minimum number of 1-factors. Discrete Math. 310 (2010), 687-691.

[8] R. K. Guy, Unsolved problems in number theory, Third edition. Problem Books in Mathematics, (Springer-Verlag, 2004).

[9] S.-G. Hwang, A note on system of distinct representatives. Kyungpook Math. J. 35 (1996), $513-516$.

[10] L. Lovász and M. D. Plummer, On bicritical graphs. in Infinite and finite sets (Colloq., Keszthely, 1973; dedicated to P. Erdös on his 60th birthday), Vol. II, Colloq. Math. Soc. Janos Bolyai 10, (North-Holland, 1975), 1051-1079.

[11] L. Lovász and M. D. Plummer, Matching Theory. North-Holland Mathematics Studies 121, Annals of Discrete Mathematics 29, (North-Holland Publishing Co.; Akadémiai Kiadó, 1986), xxvii+544 pp.

[12] B. D. McKay, Isomorph-free exhaustive generation. J. Algorithms 26 (1998), 306-324.

[13] B. D. McKay, nauty User's Guide (v. 2.4), Dept. Computer Science, Austral. Nat. Univ. (2006).

[14] S. Micali and V. V. Vazirani, An $O\left(V^{1 / 2} E\right)$ algorithm for finding maximum matching in general graphs, Proc. 21st Symp. on Foundations of Computer Science (1980), 17-27.

[15] P. A. Ostrand, Systems of distinct representatives, II. J. Math. Anal. Appl. 32 (1970), 1-4.

[16] J. Radhakrishnan, An entropy proof of Brègman's theorem. J. Combin. Theory Ser. A 77 (1997), 161-164.

[17] D. Stolee, Generating p-extremal graphs, preprint.

[18] W. T. Tutte, The factorization of linear graphs. J. London Math. Soc. 22 (1947), 107-111.

[19] L. G. Valiant, The complexity of computing the permanent. Theoret. Comput. Sci. 8 (1979), 189-201

[20] Q. R. Yu and G. Liu, Graph factors and matching extensions. (Higher Education Press, Beijing; Springer-Verlag, Berlin, 2009), xii+353 pp. 